NASA/TM-1999-209051

N. 14

Simultaneous Optical Measurements of Axial and Tangential Steady-State Blade Deflections

Anatole P. Kurkov

Glenn Research Center, Cleveland, Ohio

Harbans S. Dhadwal

Integrated Fiber Optic Systems, Inc., Stony Brook, New York

Prepared for the Turbo Expo '99

sponsored by the American Society of Mechanical Engineers

Indianapolis, Indiana, June 7-10, 1999

National Aeronautics and

Space Administration

Glenn Research Center 


\section{Acknowledgments}

The authors wish to acknowledge David Nawrocki and John Ramsey for the design of the probe actuator, Ralph Fekete for the machining; and the management, operations and installation personnel associated with the wind-tunnel test for accommodating optical instrumentation requirements.

Pratt \& Whitney is acknowledged for supplying predicted coordinates for comparison with measurements.

Available from

NASA Center for Aerospace Information 7121 Standard Drive

Hanover, MD 21076

Price Code: A03
National Technical Information Service 5285 Port Royal Road Springfield, VA 22100

Price Code: A03 


\title{
SIMULTANEOUS OPTICAL MEASUREMENTS OF AXIAL AND TANGENTIAL STEADY-STATE BLADE DEFLECTIONS
}

\author{
Anatole P. Kurkov \\ National Aeronautics and Space Administration \\ Glenn Research Center \\ Cleveland, Ohio 44135 \\ Tel:(216) 433-5695, fax:(216) 977-7051, e-mail:kurkov@grc.nasa.gov \\ Harbans S. Dhadwal \\ Integrated Fiber Optic Systems, Inc. \\ 25 E. Loop Road \\ Stony Brook, New York 11790-3350 \\ Tel:(516) 632-9537, fax:(516) 632-9537, e-mail:dhadwal @ sbee.sunysb.edu
}

\begin{abstract}
Currently, the majority of fiber-optic blade instrumentation is being designed and manufactured by aircraft-engine companies for their own use. The most commonly employed probe for optical blade deflection measurements is the spot probe. One of its characteristics is that the incident spot on a blade is not fixed relative to the blade, but changes depending on the blade deformation associated with centrifugal and aerodynamic loading. While there are geometrically more complicated optical probe designs in use by different engine companies, this paper offers an alternate solution derived from a probe-mount design feature that allows one to change the probe axial position until the incident spot contacts either a leading or a trailing edge. By tracing the axial position of either blade edge one is essentially extending the deflection measurement to two dimensions, axial and tangential. The blade deflection measurements were obtained during a wind tunnel test of a fan prototype.
\end{abstract}

\section{INTRODUCTION}

Case mounted fiber-optic sensors have been in use by airplane engine manufacturers mainly to monitor blade vibration in fans and compressors. The simplest probe arrangement is a spot probe where typically, a center fiber transmits the laser light, and the outer fibers collect the reflected light from the blade tips and transmit it to a photo-detector. Because the spot of incident light is fixed in space, whereas the blade deflects dynamically, the reflected light will originate from slightly different portions of the blade tip under different operating conditions. Unless corrections are developed to compensate for this effect, some error in vibratory tangential amplitude will occur. For the purpose of monitoring, this error is usually not critical.
This paper, however, is concerned with measuring steady-state blade deflections where it is very important to fix the spot on a blade tip at a particular location, because the deflections at a higher speed are evaluated against a low speed reference run. The change in speed usually implies a significant change in the blade orientation and possibly its shape brought about by the aerodynamic and centrifugal loading.

It is most convenient to select blade leading and trailing edges as the fixed points on a blade for which deflections will be evaluated. In order to capture blade edges at various speeds, the light probe must be movable. This was achieved by mounting the probe in an eccentric hole in a bushing that fits the fan case in the region that overlaps a blade edge path. The probe is actuated in a search for a blade edge while all the blades are viewed on an oscilloscope. The blade edge is considered captured when a pulse associated with a particular blade is significantly reduced in magnitude but is clearly distinguishable from the background noise level.

The optical probe and the electronic system were described by Dhadwal et al. (1996). The probe is constructed using integrated fiber optic technology and is capable of spatial resolution of $50 \mu \mathrm{m}$, and its outer diameter is $3.175 \mathrm{~mm}(0.125$ in.). This probe and the associated electronics were tested in the vacuum spin rig facility (Dhadwal and Kurkov, 1998).

The steady-state tangential blade deflections can be measured by an optical line probe (Chi et. al., 1987) which has been considerably improved by engine manufacturers. The line probe, however, is optically more complicated and its resolution is not as good as those obtainable with current spot probes. The other alternative is to use a series of spot probes capable of mapping suction and pressure surfaces of a blade tip. This method would require considerable software development and is also costly. 


\section{PROBE ACTUATORS}

Figure 1 shows a cross-section of a probe actuator mounted in the casing. It includes a probe with a fiber optic cable attached to its end. The probe is positioned in the rotating casing-plug eccentrically with an of fset of $9.14 \mathrm{~mm}(0.36 \mathrm{in}$.) from the axis of the plug. The tip of a fiber optic probe is flush with the lower surface of the plug. The probe is sealed in the plug with a rubber o-ring (not shown in the assembly drawing) and secured with a setscrew (visible in the upper ring that guides the probe through aluminum housing). When installed in the casing, the face of the bushing is recessed about $0.5 \mathrm{~mm}(0.020 \mathrm{in}$.) from the rub strip. Underneath the bushing flange, a lower teflon washer provides a low-friction seal for the casing port. The teflon washer on the upper side of the flange together with the spring washer above it provide a tight sliding interface between the rotating flange and the stationary aluminum housing. The spring washer shown with non-hatched cross-section, actually consists of a rubber o-ring, and another teflon washer above it, which contacts the aluminum housing. Thus, in the assembly the rubber o-ring is sandwiched between the two teflon washers. The function of the o-ring is to provide a spring force and to evenly distribute it. Originally, in its place, a wavy spring washer was used: however, the current arrangement results in a more consistent performance and a lower friction force for a given normal force.

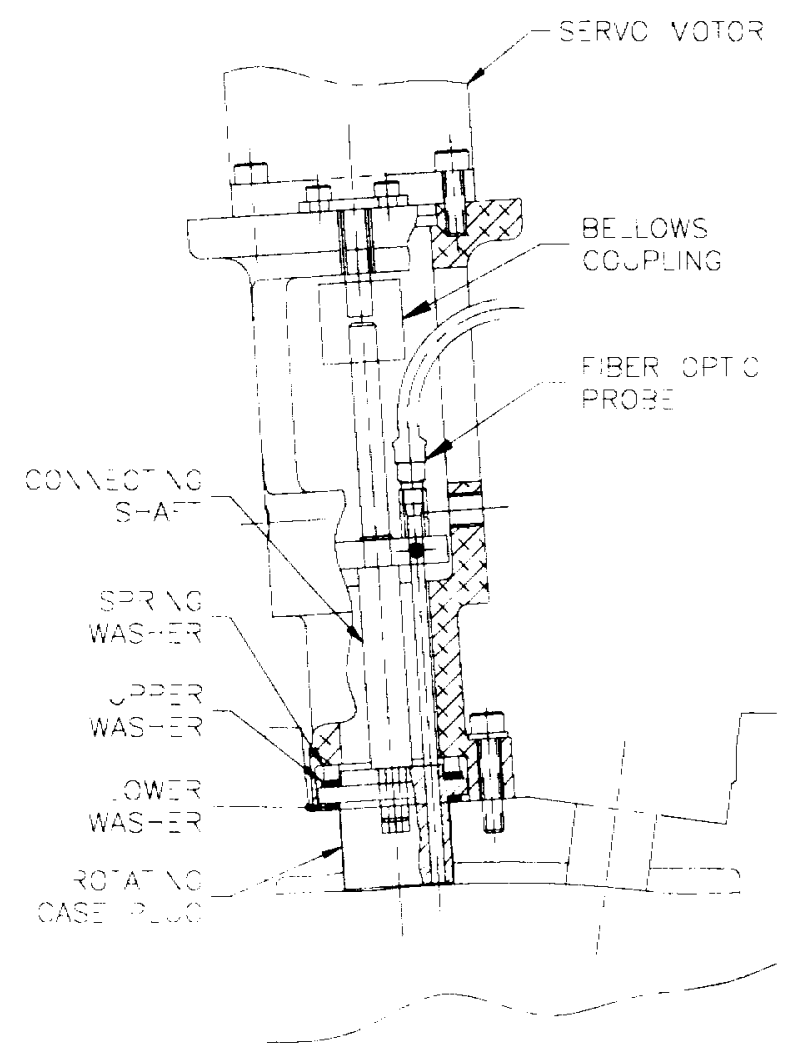

Fig. 1 Actuator assembly drawing
A $6.35 \mathrm{~mm}(0.25 \mathrm{in})$ shaft is attached to the bushing and joined through a flexible bellows coupling to a servomotor shaft. As shown, the cylindrical enclosure is open on one side in order to provide an exit path for the fiber optic cable. The 180 degrees opening in the housing is oriented such that its base (along the diameter) is parallel to the fan rotor axis. Thus, when the motor is actuated, the probe moves over semi-circular path, maximizing the extent of the motion in the axial direction. The two non-contacting limit switches (not shown in the figure) restrict the extent of rotation to 180 degrees. The servomotor had a resolution of 4000 counts per revolution and it was remotely controlled by a computer. Figure 2 is a photograph of the servomotor-actuator assembly.

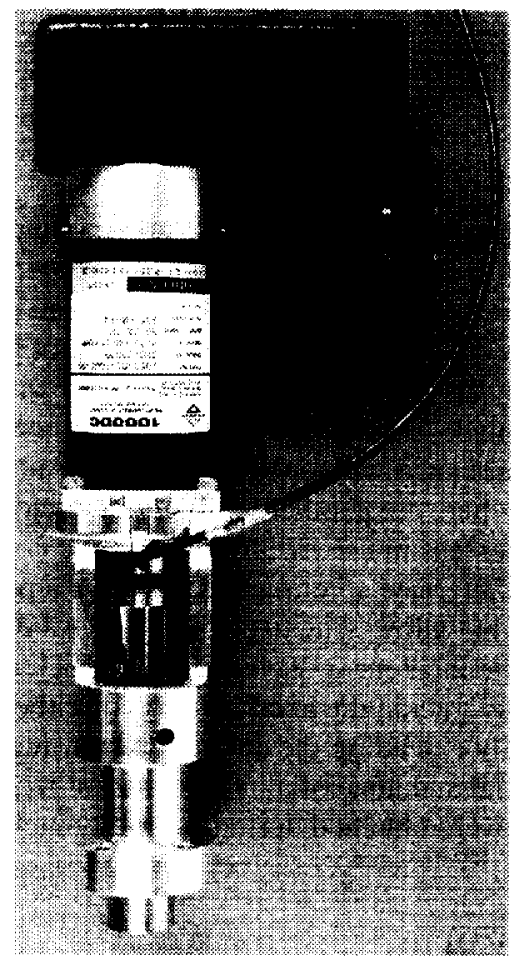

Fig. 2 Actuator and motor assembly

\section{BLADE DEFLECTION MEASUREMENTS}

The steady-state blade deflections were measured during a fan prototype acoustic test in the $9 \times 15$ feet low speed wind tunnel at Glenn Research Center. The $0.5588 \mathrm{~m}$ (22 in.) diameter, 18 blade fan rotor was driven by an air turbine mounted in the rear of the rig. The fan rig was equipped with the bellmouth followed by the inlet duct. The two probe actuators were mounted in view of the leading and trailing edge trajectories.

Figure 3 shows locations of the leading and trailing edge probe actuators and the relative location of the blade chord at the time the leading edge of a blade is being sensed by the probe at 1000 $\mathrm{rpm}$ (solid line, $C_{1}$ ). The speed for the dashed line in Fig. 3 was 
$7950 \mathrm{rpm}\left(C_{2}\right)$. The two circles indicate possible probe positions when actuators are set in motion about the centerline of the respective ports. The indicated radii at the two ports define the probe positions necessary to capture the leading and trailing edges associated with the $C_{1}$ chord line. At the instance depicted in the figure, the blade leading edge is being sensed by its probe; however, the trailing edge will be sensed somewhat later, when it traverses the distance indicated by the dotted line (from TE probe to TE of $C_{1}$ ).

All the timing information necessary for determination of blade deflections is obtained from oscilloscope traces of blade pulses at the time when a blade edge is being sensed. However, prior to the search for blade edges, the full blade pulses were captured with actuators positioned to intersect all blades at some small distance away from blade edges. Figures 4 and 5 illustrate full blade amplitudes for the leading edge for 1000 and $7950 \mathrm{rpm}$ with respective resolutions of 2 and $0.2 \mu \mathrm{s}$. The upper speed corresponded to the design point. On the low end, the speed was restricted by the rig operational requirements. These two speeds, however, were sufficiently apart to illustrate the technique. Note that there were no synchronous vibrations were observed at these speeds.

A total of 50,000 points were captured for each trace which was the memory limit of the oscilloscope. The vertical resolution of 8 bits ( 128 levels) was divided among 4 channels displayed on the oscilloscope at one time. In selecting the time resolution on the oscilloscope the main consideration was that at each speed at the least one full revolution was captured, so that each trace contained all 18 blades. The oscilloscope was triggered at $0 \mu \mathrm{s}$ by the reference once-per-revolution pulse generated by a specially designed optical sensor capable of the same spatial resolution as the blade sensors. The length of each revolution for each trace was determined by number of counts between two identical blade pulses.

During a traverse toward a blade edge the pulses become narrower and the amplitude for some blade decreases. (Note that the traces for all blades are not identical because of the blade-to-blade differences). Upon continuing the traverse in the same direction, some of the blades with lower amplitudes start disappearing from the trace. Typically, several traces at each speed were obtained until a substantial number of blades vanished. Each of these traces defines leading or a trailing edge axial position for some blades. A blade edge is captured when its pulse height becomes appreciably reduced relative to the full pulse amplitude captured prior to the traverse. However, the edge pulse amplitude at this instance should be sufficiently above the signal noise floor and the pulse slopes should be well defined.

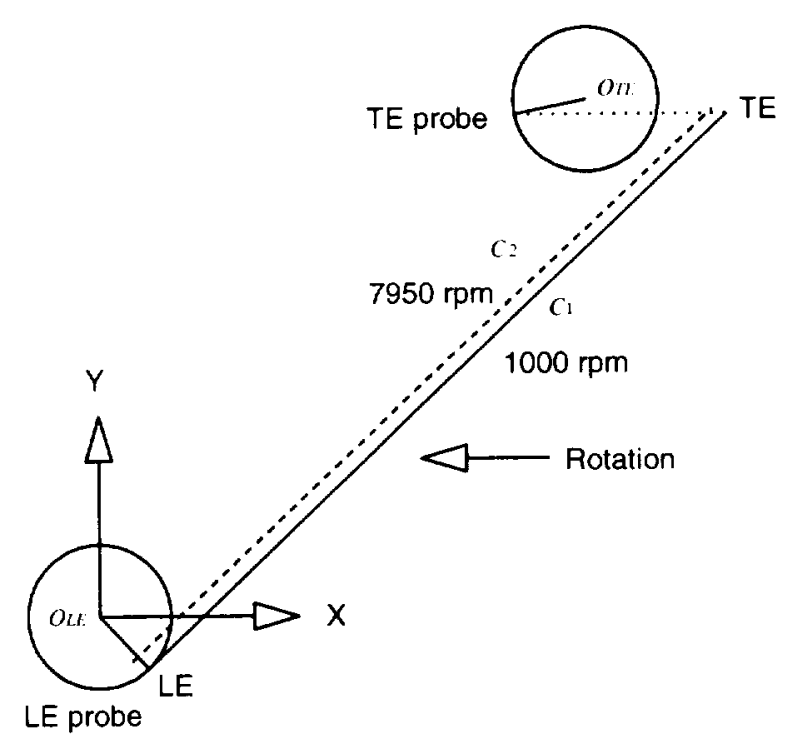

Fig. 3 Probe configuration for edge detection

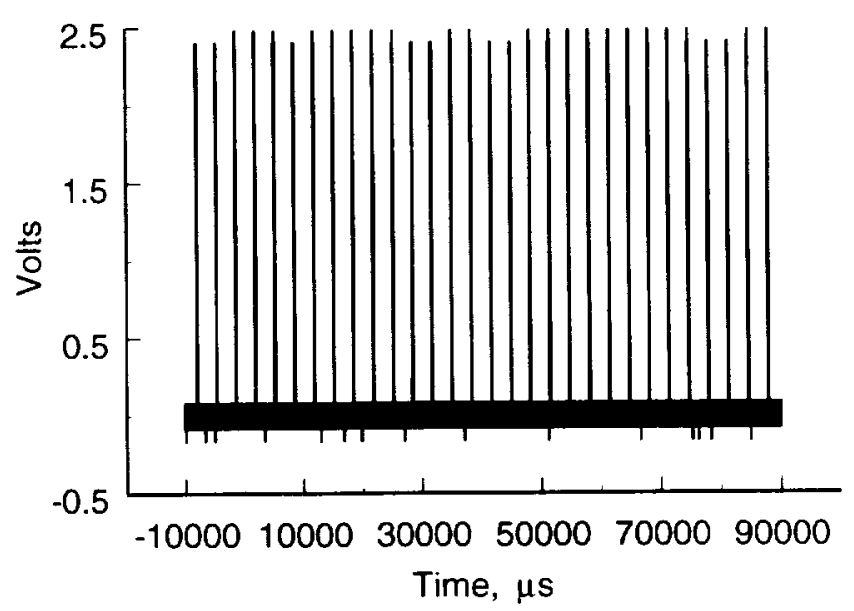

Fig. 4 Blade pulses near LE, 1000 rpm

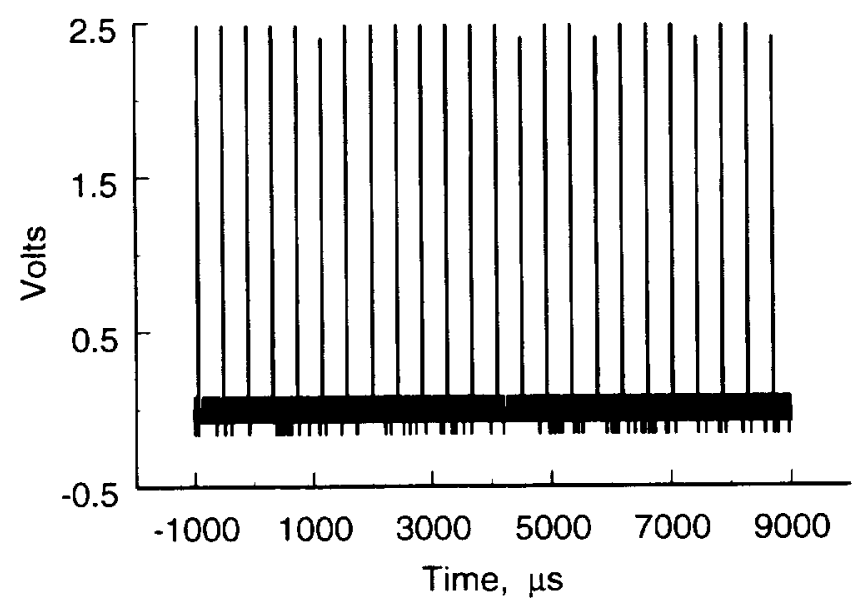

Fig. 5 Blade pulses near LE, 7950 rpm 
Figures 6 and 7 illustrate the traces that were selected to define leading edges at the 1000 and $7950 \mathrm{rpm}$ for blades 7 and 18 , counting from first blade following the trigger point 0 .

Corresponding traces were also available for these blades at the trailing edge.

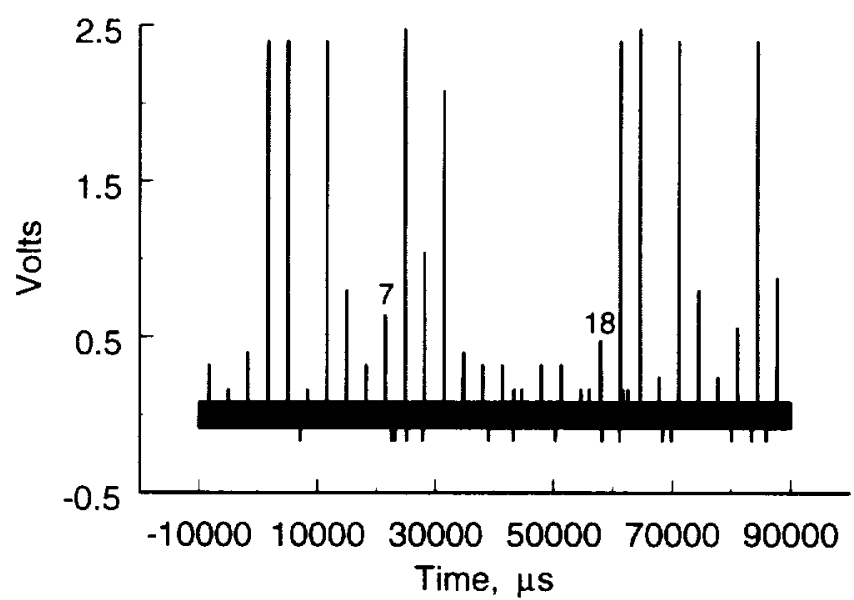

Fig. 6 Blade pulses during LE detection, 1000 rpm

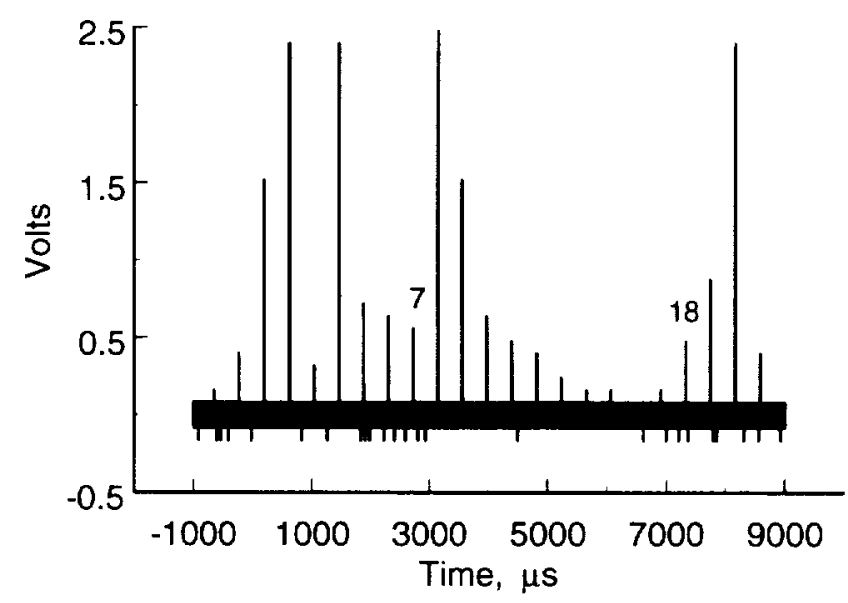

Fig. 7 Blade pulses during LE detection, 7950 rpm

Figures 8 and 9 single-out blades 7 and 18 and indicate selected blades trigger points. The non-uniformity of the axial blade positions is clearly illustrated in Fig. 10 for the trailing edge when, essentially, only one blade pulse remained (corresponding to blade 15). Figure 11 illustrates the pulse shape for this blade indicating that the beam has not yet reached the edge, since there is some flatness remaining at the tip. The $\mathrm{X}$ and $\mathrm{Y}$ coordinates of the leading edge were obtained from the recorded angular position of the probe and the known radius of the probe eccentric. The $X$ and $Y$ coordinates of the trailing edge are computed taking into account the offset of the trailing edge actuator axis $\left(\mathrm{O}_{\mathrm{TE}}\right)$ relative to the reference leading edge axis $\left(\mathrm{O}_{\mathrm{LF}}\right)$. In addition, for the $\mathrm{X}$ coordinate, the distance corresponding to the dashed line connecting TE probe to TE must be known. This distance is computed from the measured time delay between the leading and trailing edge pulses captured on the oscilloscope and the known fan speed associated with the trace.

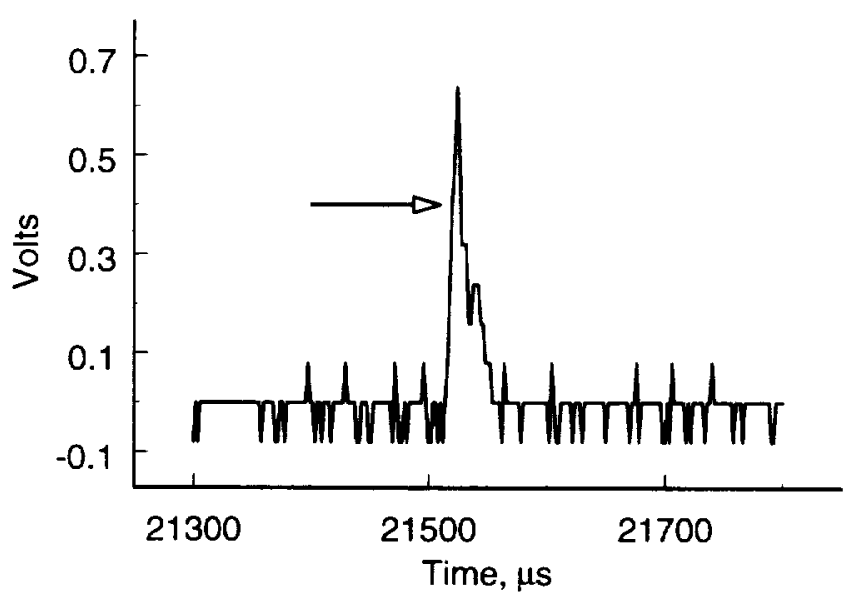

Fig. 8 Trigger point for blade 7, 1000 rpm

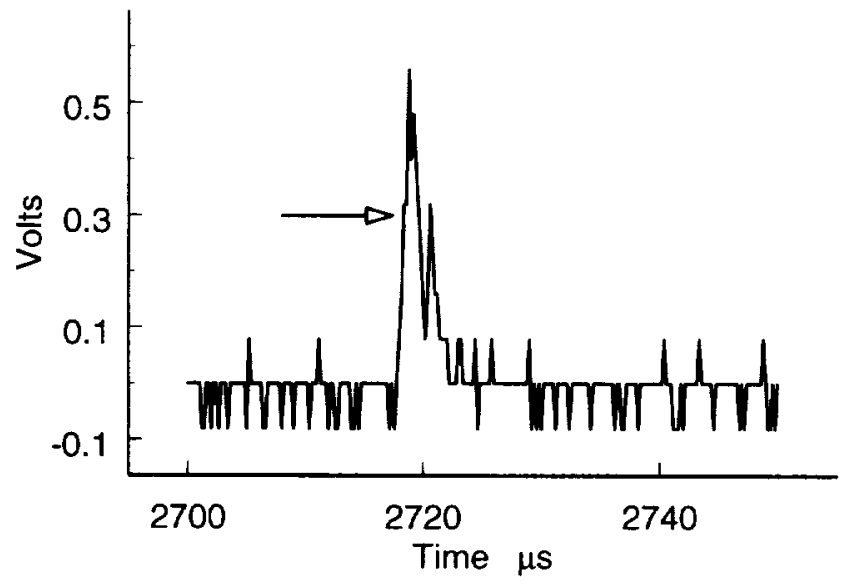

Fig. 9 Trigger point for blade 7, 7950 rpm

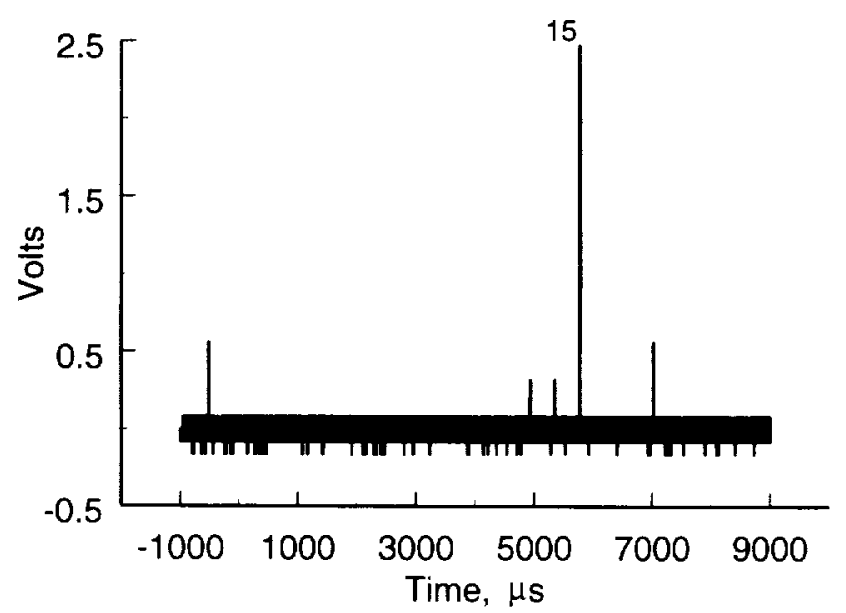

Fig. 10 TE trace with only one full amplitude blade pulse 


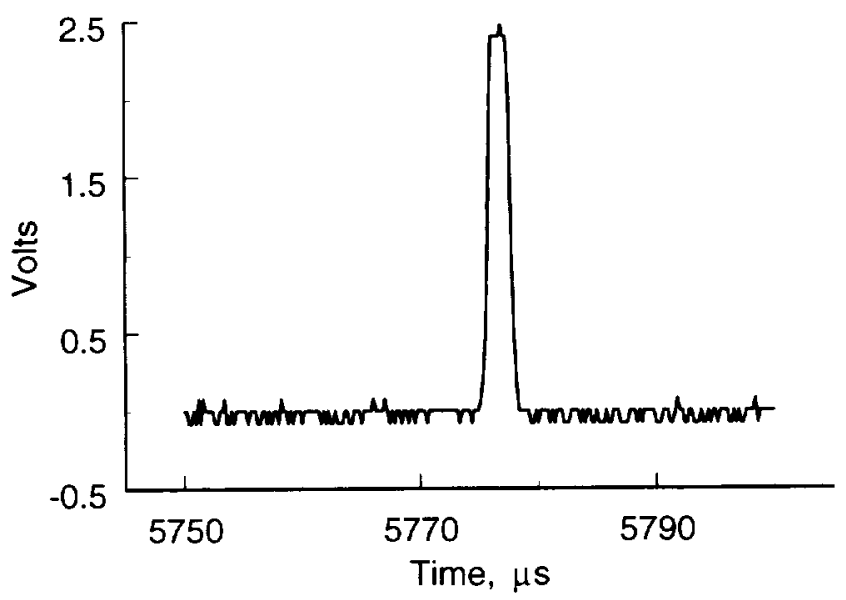

Fig. 11 Detail of blade 15, TE, $7850 \mathrm{rpm}$

The total displacements at $7950 \mathrm{rpm}$ relative to $1000 \mathrm{rpm}$ are presented in Table 1. Since the available oscilloscope records indicated that blades 7 and 18 were captured at these speeds on the same traces, for both leading and trailing edges, these two blades must have the same axial displacements. The values in Table 1 can be converted to angular units by using fan rotor diameter, which was $55.88 \mathrm{~cm}$ (22 in).

Table 1 Total displacements

\begin{tabular}{|c|c|c|c|}
\hline \multicolumn{2}{|c|}{ Total displacement } & \multicolumn{2}{|c|}{$[\mathrm{mm}($ in)] } \\
\cline { 3 - 4 } \multicolumn{2}{|c|}{} & Blade 7 & Blade 18 \\
\hline \multirow{2}{*}{$\begin{array}{c}\text { Leading } \\
\text { edge }\end{array}$} & $\Delta \mathrm{X}$ & $-2.074(-0.0817)$ & $-2.070(-0.0815)$ \\
\cline { 2 - 4 } & $\Delta \mathrm{Y}$ & $0.982(0.0387)$ & $0.982(0.0387)$ \\
\hline \multirow{2}{*}{$\begin{array}{c}\text { Trailing } \\
\text { edge }\end{array}$} & $\Delta \mathrm{X}$ & $-1.825(-0.0718)$ & $-1.749(-0.0689)$ \\
\cline { 2 - 4 } & $\Delta \mathrm{Y}$ & $0.538(0.0212)$ & $0.538(0.0212)$ \\
\hline
\end{tabular}

The rotational displacements of the chord line for these two blades are presented in Table 2 . The blade chord vector is defined as

$$
\vec{C}=\vec{R}_{T E}-\vec{R}_{L E}
$$

where subscripts TE and LE denote the end points of position vectors that start at $\mathrm{O}_{\mathrm{LE}}$ (Fig. 12). The rotation of the chord vector, $\theta$, relative to $1000 \mathrm{rpm}$ is then computed from

$$
\sin \theta=\frac{\overrightarrow{\mathrm{C}}_{1} \times \overrightarrow{\mathrm{C}}_{2}}{\left|\overrightarrow{\mathrm{C}}_{1}\right| \cdot\left|\overrightarrow{\mathrm{C}}_{2}\right|}
$$

where subscripts 1 and 2 correspond to 1000 and $7950 \mathrm{rpm}$.
Table 2 Rotational displacements

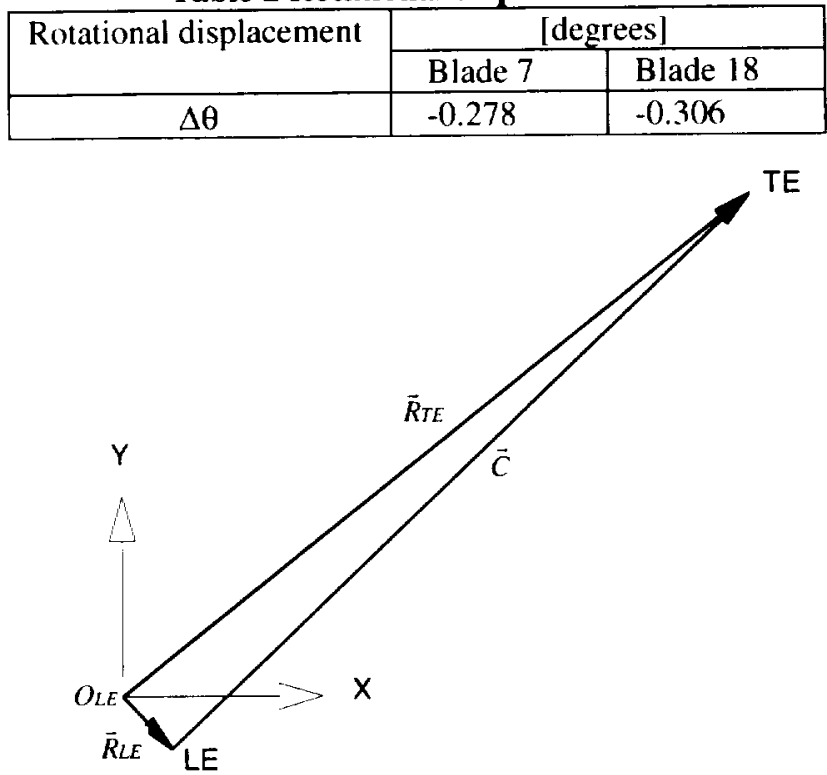

Fig. 12 Chord vector diagram

Blade 7 in Figs. 6 and 7 indicates a typical amplitude level chosen to define a blade edge. In order to establish the sensitivity of results to the edge amplitude choice, one can select somewhat different edge amplitude. For example, Fig. 13 illustrates the leading edge amplitudes at $1000 \mathrm{rpm}$ corresponding to the leading edge eccentric position of -48.69 degrees rather than -46.8 degrees as in Fig. 6 (a difference of -1.89 degrees). Had the amplitude of blade 7 in Fig. 13 (rather than in Fig. 6) been chosen to signify the presence of the LE, the corresponding axial deflection would increase in magnitude by $0.2 \mathrm{~mm}(0.008 \mathrm{in})$ from the value given in Table 1 .

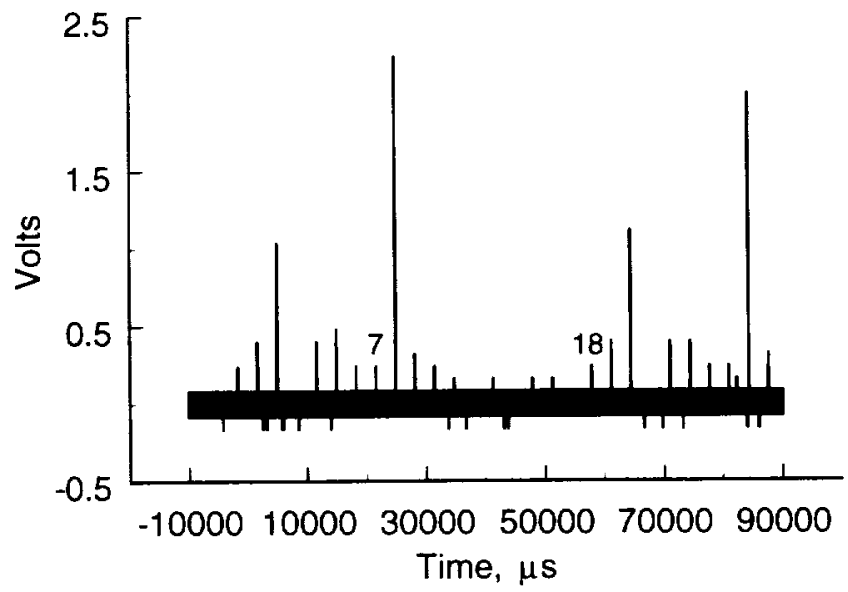

Fig. 13 Blade pulse trace corresponding to eccentric position $-\mathbf{1 . 8 9}$ degrees relative to Fig. 6 
However, since the amplitudes that were taken to define the presence of a LE or a TE were in general within half the amplitude difference for blades 7, depicted in Figs 6 and 13, the error is more likely to be within $0.1 \mathrm{~mm}(0.004 \mathrm{in})$. This error is somewhat greater than the resulting spatial resolution obtained with the oscilloscope for the given sampling speed, which was $0.058 \mathrm{~mm}(0.0023 \mathrm{in})$.

The triggering consistency can be improved by acquiring data more frequently during a LE or a TE search and by selecting an oscilloscope that has better vertical resolution than the current oscilloscope. An improvement in resulting spatial resolution can be achieved with an oscilloscope that has a deeper memory.

Another possible source of error can occur if there is an axial motion of the fan-rotor shaft, as a result of the pressure differential across rotor and the existing clearances in the thrust bearing. For this reason, an eddy current proximity probe was installed in the wind tunnel rig to measure the axial displacement of the shaft. The probe was calibrated in the spin rig using a special rotor that reproduced the wind-tunnel sensing-tooth geometry. The lengths of the cables that were used in the wind tunnel were also matched during the calibration. The shaft axial displacement at $4460 \mathrm{rpm}$ relative to $1000 \mathrm{rpm}$ was less than $0.0254 \mathrm{~mm}(0.001 \mathrm{in})$. Unfortunately, this measurement was not recorded at $7950 \mathrm{rpm}$; however, based on the results obtained at $4460 \mathrm{rpm}$ one can expect it to be very small and within the axial blade displacements measurement accuracy.

Figure 14 presents computed blade-tip, mid-thickness $\mathrm{X}$ and $\mathrm{Y}$ coordinates at 1000 and $7950 \mathrm{rpm}$ along with measured LE and TE positions computed by adding the measured relative $X$ and $Y$ displacements to the respective predicted coordinates at 1000 $\mathrm{rm}$. On the scale of this figure, the two points from Table lare indistinguishable. Predicted coordinates were obtained by $R$. Neubert and W. Owen-Peer of Pratt and Whitney. Both, inertial and aerodynamic loads were included in predictions.

Apparently. the steady-state blade deflections at these speeds were very small.

Predicted and measured displacements at the leading and trailing edges at $7950 \mathrm{rpm}$ relative $101000 \mathrm{rpm}$ are presented in Fig. 15. For these two blades, the data appears to be consistent; however, they were captured at the same instant and, therefore. their axial displacements are the same. (Note that the leading edge points are nearly coincident.) On the other hand, blade to blade variability in the axial blade positions at the leading edge, for example. was typically about $0.5 \mathrm{~mm}$, which is of the same order as the difference between measured and predicted displacements. Because of this, for a rigorous quantitative analysis, one would have to look at the history of more than just two blades.

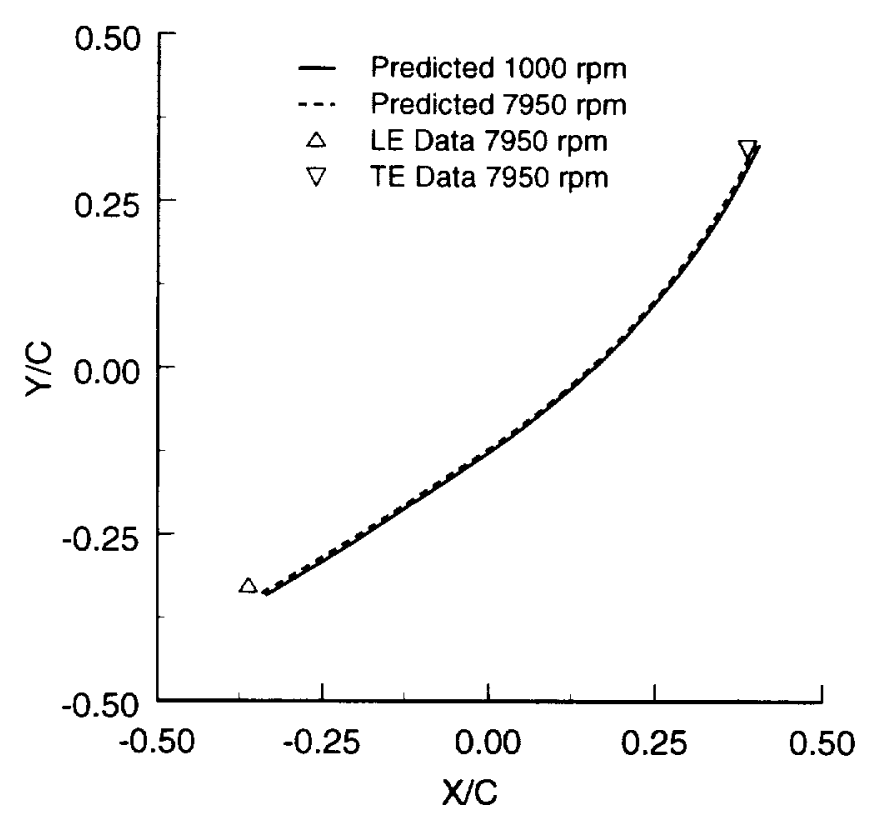

Fig. 14 Predicted mid-thickness blade-tip section coordinates at 1000 and $7950 \mathrm{rpm}$. Data points at the $L E$ and $T E$ edges at $7950 \mathrm{rpm}$ are relative to corresponding predicted $1000 \mathrm{rpm}$ coordinates.

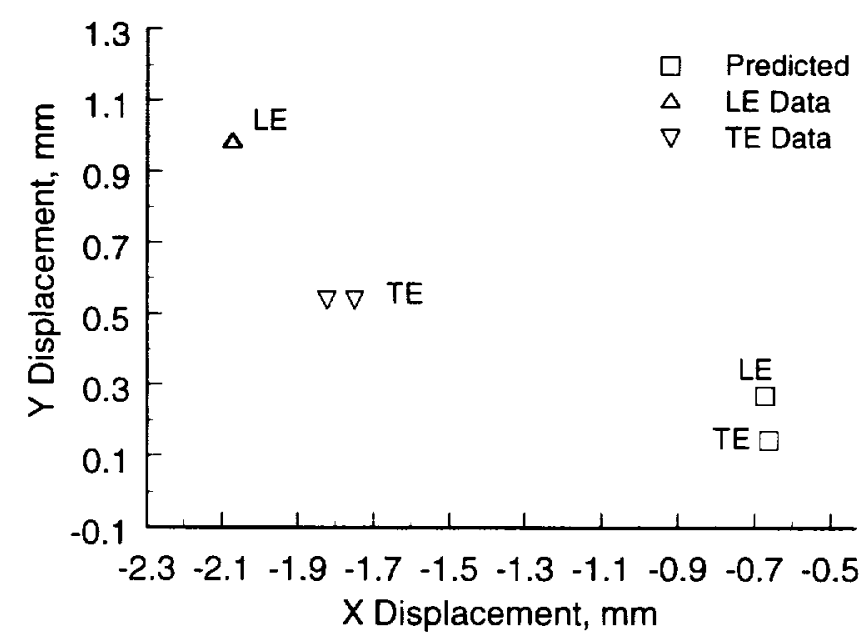

Fig. $15 \mathrm{LE}$ and TE displacements at $7950 \mathrm{rpm}$ relative to $1000 \mathrm{rpm}$. The data points correspond to blades 7 and 18. 
Predicted and measured rotational displacement are both small. They are, respectively, -0.0522 and -0.292 degrees (average of the two values in Table 2 ).

In this initial application, we restricted data collection to several discrete points at each speed. This enabled us to single out the two blades that satisfied the chosen threshold edgedetection criteria. However, a more practical procedure would be to perform a slow and controlled 180 (or lesser) degree sweep with the actuator concurrently acquiring waveform data, along with actuator angular position. An algorithm could be than applied to sort he data into revolutions and track the occurrence of blade edge encounters and the associated angular position of the actuator rotor. This provides sufficient information to determine tip clearance for each blade. Note that rather then recording data continuously, a segmented data acquisition, concurrently with the segment time stamping, could be incorporated instead. These features are currently available in some commercial wave-capture data-acquisition boards. This would conserve the memory and speed up the analyses.

\section{CONCLUDING REMARKS}

This technique is well suited for measurements of steady state relative blade deflections in axial and tangential direction. Its main advantage is that it uses standard high-resolution fiberoptic spot probes that are already developed and available. A standard oscilloscope can be used to acquire data with acceptable accuracy; however, currently the data acquisition and analyses are somewhat time consuming. Automating these procedures is possible but it would require additional effort and expense.

\section{REFERENCES}

Chi, M. R., Cutts G. D., Haas D. C., Puffer G. L. Jr., Shu H. T.. and Zepke B.E., 1987, " Demonstration Testing of a

Noninterference Stress Measurement System,"

Technical Report R87-957037-14F (USAF Contract F40600-84-C-0009), United Technologies Research Center, East Hartford, CT.

Dhadwal. H. S., Mehmud, A., Khan, R., and Kurkov, A. P., 1996, "Integrated fiber optic light probe: Measurement of static deflections in rotating turbomachinery,"

Rev. Sci. Instrum. Vol. 67 (2)

Dhadwal, H. S., and Kurkov, A. P., 1998, "Dual-Laser Probe Measurements of Blade-Tip Clearance,"

ASME paper no. 98-GT-183 


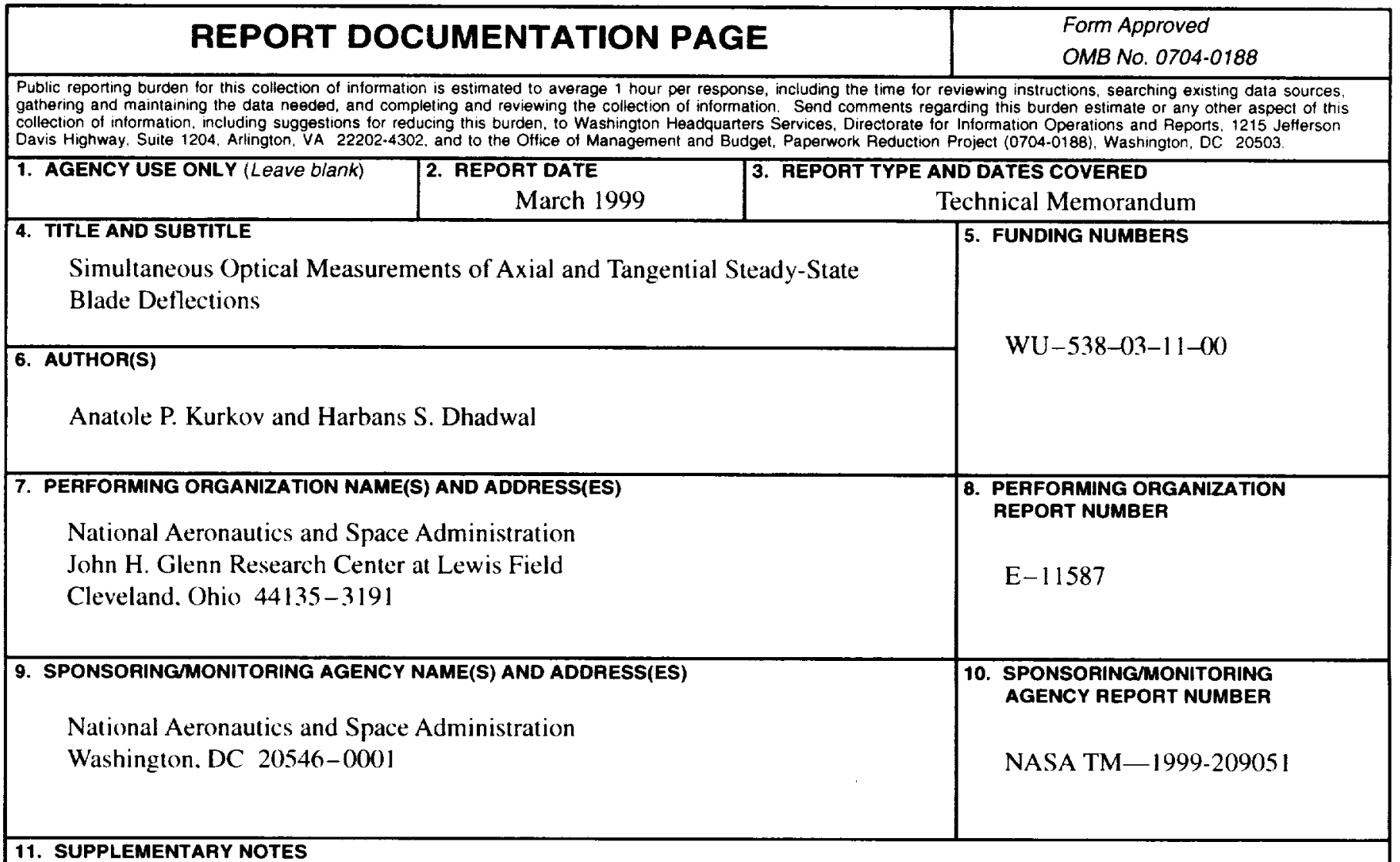

Prepared for the Turbo Expo ' 99 sponsored by the American Society of Mechanical Engineers, Indianapolis, Indiana, June 7-10. 1999. Anatole P. Kurkov, Glenn Research Center; and Harbans S. Dhadwal, Integrated Fiber Optic Systems, Inc., Stony Brook, New York 11790-3350. Responsible person, Anatole P. Kurkov, organization code 5930, (216) 433-5695.

12a. DISTRIBUTION/AVAILABILITY STATEMENT 12b. DISTRIBUTION CODE

Unclassified - Unlimited

Subject Categories: 35 and 74

Distribution: Nonstandard

This publication is available from the NASA Center for AeroSpace Information. (301) 621-0390.

13. ABSTRACT (Maximum 200 words)

Currently, the majority of fiber-optic blade instrumentation is being designed and manufactured by aircraft-engine companies for their own use. The most commonly employed probe for optical blade deflection measurements is the spot probe. One of its characteristics is that the incident spot on a blade is not fixed relative to the blade, but changes depending on the blade deformation associated with centrifugal and aerodynamic loading. While there are geometrically more complicated optical probe designs in use by different engine companies, this paper offers an alternate solution derived from a probemount design feature that allows one to change the probe axial position until the incident spot contacts either a leading or a trailing edge. By tracing the axial position of either blade edge one is essentially extending the deflection measurement to two dimensions, axial and tangential. The blade deflection measurements were obtained during a wind tunnel test of a fan prototype.

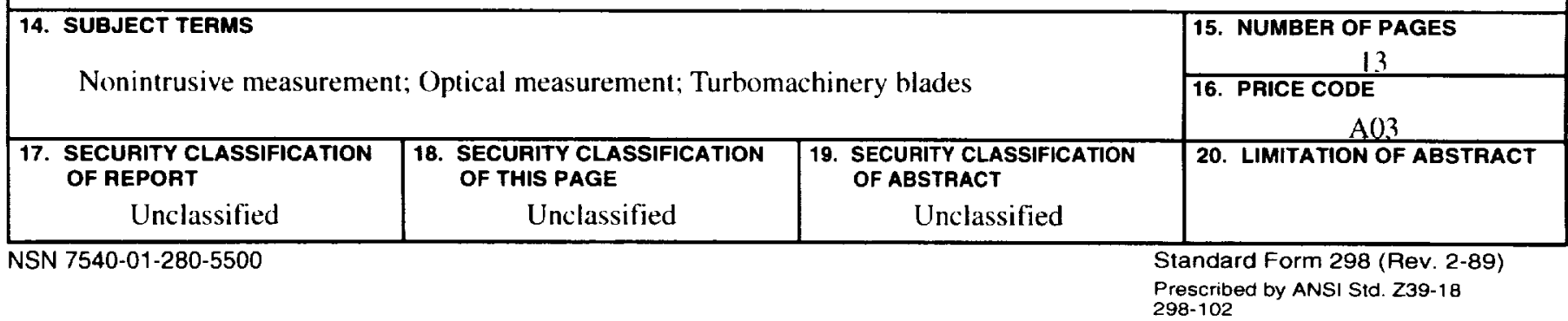

